\title{
Double controlled metric-like spaces
}

Nabil Mlaiki ${ }^{1 *}$ (10)

Correspondence:

nmlaiki@psu.edu.sa;

nmlaiki2012@gmail.com

${ }^{1}$ Department of Mathematics and

General Sciences, Prince Sultan

University, Riyadh, Saudi Arabia

11586

\begin{abstract}
In this paper, we introduce a new extension of the double controlled metric-type spaces, called double controlled metric-like spaces, by assuming that the "self-distance" may not be zero On the other hand, if the value of the metric is zero, then it has to be a "self-distance" (i.e., we replace $[\varsigma(g, h)=0 \Leftrightarrow g=h]$ by $[\varsigma(g, h)=0 \Rightarrow g=h])$. Using this new type of metric spaces, we generalize many results in the literature. We prove fixed point results along with examples illustrating our theorems. Also, we present double controlled metric-like spaces endowed with a graph along with an open question.

MSC: $47 \mathrm{H} 10 ; 54 \mathrm{H} 25$

Keywords: Fixed point; $b$-Metric spaces; Extended b-metric space; Controlled metric type spaces; Double controlled metric type spaces; Double controlled metric like spaces
\end{abstract}

\section{Introduction}

In 1922, Banach [1] proved the existence and uniqueness of a fixed point for selfcontractive mapping in metric spaces. That was the starting point for researchers in the field of analysis to generalize his result, whether by changing the contractions or by generalizing the type of metric spaces covering a wider class of metrics, for example, extension of metric spaces to partial metric spaces or $b$-metric spaces; see [2-17].

One interesting extension of metric spaces is $b$-metric spaces introduced by Bakhtin [18]. Recently, several extensions of $b$-metric spaces were introduced, such as extended $b$-metric spaces, which were initiated by Kamran et al. [19]. In 2018, Mlaiki et al. [20] gave an extension of the extended $b$-metric spaces, the so-called controlled metric-type spaces. Also, in 2018, Abdeljawad et al. [21], introduced the concept of double controlled metrictype spaces. As an extension of all the types of metric spaces mentioned, we introduce a new class, the so-called double controlled metric-like spaces.

\section{Preliminaries}

We begin our preliminaries by recalling the definition of extended $b$-metric spaces.

Definition 2.1 ([19]) Consider the set $\mathcal{F} \neq \emptyset$ and a function $\hbar: \mathcal{F} \times \mathcal{F} \rightarrow[1, \infty)$. Suppose that a function $\varsigma: \mathcal{F} \times \mathcal{F} \rightarrow R^{+}$satisfies the following conditions for all $g, h, w \in \mathcal{F}$ :

(1) $\varsigma(g, h)=0 \Longleftrightarrow g=h$;

(c) The Author(s) 2020. This article is licensed under a Creative Commons Attribution 4.0 International License, which permits use sharing, adaptation, distribution and reproduction in any medium or format, as long as you give appropriate credit to the original author(s) and the source, provide a link to the Creative Commons licence, and indicate if changes were made. The images or other third party material in this article are included in the article's Creative Commons licence, unless indicated otherwise in a credit line to the material. If material is not included in the article's Creative Commons licence and your intended use is not permitted by statutory regulation or exceeds the permitted use, you will need to obtain permission directly from the copyright holder. To view a copy of this licence, visit http://creativecommons.org/licenses/by/4.0/. 
(2) $\varsigma(g, h)=\varsigma(h, g)$;

(3) $\varsigma(g, h) \leq \hbar(g, h)[\varsigma(g, w)+\varsigma(w, h)]$.

Then the pair $(\mathcal{F}, \varsigma)$ is called an extended $b$-metric space.

Next, we present the definition of controlled metric-type spaces.

Definition 2.2 ([20]) Given a nonempty set $\mathcal{F}$ and a function $\varpi: \mathcal{F}^{2} \rightarrow[1, \infty)$, suppose that a function $\rho: \mathcal{F}^{2} \rightarrow[0, \infty)$ satisfies the following conditions for all $g, h, w \in \mathcal{F}$ :

$\left(\rho_{1}\right) \rho(g, h)=0 \Leftrightarrow g=h$;

$\left(\rho_{2}\right) \rho(g, h)=\rho(h, g)$;

$\left(\rho_{3}\right) \rho(g, h) \leq \varpi(g, w) \rho(g, w)+\varpi(w, h) \rho(w, h)$.

Then the pair $(\mathcal{F}, \rho)$ is called a controlled metric-type space.

The following definition is a generalization of controlled metric-type spaces to double controlled metric-type spaces.

Definition 2.3 ([21]) (DCMTS) Consider a set $\mathcal{F} \neq \emptyset$ and noncomparable functions $\varpi, \varepsilon: \mathcal{F} \times \mathcal{F} \rightarrow[1, \infty)$. Suppose that a function $\varsigma: \mathcal{F}^{2} \rightarrow[0, \infty)$ satisfies the following conditions for all $g, h, w \in \mathcal{F}$ :

(1) $\varsigma(g, h)=0$ if and only if $g=h$;

(2) $\varsigma(g, h)=\varsigma(h, g)$;

(3) $\varsigma(g, h) \leq \varpi(g, w) \varsigma(g, w)+\varepsilon(w, h) \varsigma(w, h)$.

Then the pair $(\mathcal{F}, \varsigma)$ is called a double controlled metric-type space.

Now we present our generalization of the double controlled metric-type spaces.

Definition 2.4 (DCMLS) Consider a set $\mathcal{F} \neq \emptyset$ and noncomparable functions $\varpi, \varepsilon: \mathcal{F} \times$ $\mathcal{F} \rightarrow[1, \infty)$. Suppose that a function $\varsigma: \mathcal{F} \times \mathcal{F} \rightarrow[0, \infty)$ satisfies the following conditions for all $g, h, w \in \mathcal{F}$ :

$\left(\varsigma_{1}\right) \varsigma(g, h)=0 \Rightarrow g=h$;

$\left(\varsigma_{2}\right) \varsigma(g, h)=\varsigma(h, g)$;

$\left(\varsigma_{3}\right) \varsigma(g, h) \leq \varpi(g, w) \varsigma(g, w)+\varepsilon(w, h) \varsigma(w, h)$.

Then the pair $(\mathcal{F}, \varsigma)$ is called a double controlled metric-like space $(D C M L S)$.

We denote double controlled metric-type spaces by (DCMTS) and double controlled metric-like spaces by $(D C M L S)$.

Remark 2.5 Note that any $(D C M T S)$ is a $(D C M L S)$. However, the converse is not always true.

Example 2.6 Let $\mathcal{F}=\mathbb{R}^{+}$. Take $\varsigma: \mathcal{F} \times \mathcal{F} \rightarrow \mathbb{R}^{+}$defined as

$$
\varsigma(g, h)= \begin{cases}0 & \Rightarrow g=h, \\ \frac{1}{2} & \text { if } g=0 \text { and } h=0 \\ \frac{1}{g} & \text { if } g \geq 1 \text { and } h \in[0,1) \\ \frac{1}{h} & \text { if } h \geq 1 \text { and } g \in[0,1) \\ 1 & \text { otherwise. }\end{cases}
$$


Consider the following $\varpi, \varepsilon: \mathcal{F} \times \mathcal{F} \rightarrow[1, \infty)$ :

$$
\varpi(g, h)=\left\{\begin{array}{ll}
g & \text { if } g, h \geq 1, \\
1 & \text { otherwise, }
\end{array} \text { and } \varepsilon(g, h)= \begin{cases}1 & \text { if } g, h<1 \\
\max \{g, h\} & \text { otherwise }\end{cases}\right.
$$

It is not difficult to see that $\left(\varsigma_{1}\right)$ and $\left(\varsigma_{2}\right)$ are satisfied. Next, we show that condition $\left(\varsigma_{3}\right)$ is satisfied.

Case 1: If $w=g$ or $w=h$, then $\left(\varsigma_{3}\right)$ is satisfied.

Case 2: If $w \neq g$ and $w \neq h$, note that if $g=h$, then we are done. So, without loss of generality, we may assume that $g \neq h$. Thus $g \neq h \neq w$. It is not difficult to see that if ( $g \geq 1$ and $0 \leq h<1)$ or $(h \geq 1$ and $0 \leq g<1)$, then $\left(\varsigma_{3}\right)$ holds. Now we consider the following subcases:

Subcase 1: $g, h \geq 1$.

If $w \geq 1$, then we are done. Also, if $0 \leq w<1$, then we get

$$
1 \leq \frac{1}{g}+h \cdot \frac{1}{h}
$$

and hence $\left(\varsigma_{3}\right)$ holds.

Subcase 2: $g, h<1$.

If $0 \leq w<1$, then we are done. On the other hand, if $w \geq 1$, then we have

$$
1 \leq \frac{1}{w}+w \cdot \frac{1}{w}
$$

and thus $\left(\varsigma_{3}\right)$ is satisfied. Therefore $(\mathcal{F}, \varsigma)$ is a $(D C M L S)$.

Moreover,

$$
\varsigma(0,0) \neq 0,
$$

and thus $(\mathcal{F}, \varsigma)$ is not a $(D C M T S)$.

Example 2.7 Let $\mathcal{F}=\{0,1,2\}$. Define $\varsigma$ by

$$
\varsigma(0,0)=\varsigma(1,1)=0, \quad \varsigma(2,2)=\frac{1}{10},
$$

and

$$
\varsigma(0,1)=\varsigma(1,0)=1, \quad \varsigma(0,2)=\varsigma(2,0)=\frac{1}{2}, \quad \varsigma(1,2)=\varsigma(2,1)=\frac{2}{5} .
$$

Take $\varpi, \varepsilon: \mathcal{F} \times \mathcal{F} \rightarrow[1, \infty)$ to be symmetric and defined by

$$
\varpi(0,0)=\varpi(1,1)=\varpi(2,2)=\varpi(0,2)=1, \quad \varpi(1,2)=\frac{5}{8}, \quad \varpi(0,1)=\frac{11}{10},
$$

and

$$
\varepsilon(0,0)=\varepsilon(1,1)=\varepsilon(2,2)=1, \quad \varepsilon(0,2)=\frac{3}{2}, \quad \varepsilon(1,2)=\frac{5}{4}, \quad \varepsilon(0,1)=\frac{11}{10} .
$$


Note that $(\mathcal{F}, \varsigma)$ is a $(D C M L S)$. Also, we have;

$$
\varsigma(2,2)=\frac{1}{10} \neq 0 .
$$

Thus $(\mathcal{F}, \varsigma)$ is not a $(D C M T S)$.

Next, we present the topology of the controlled metric-like spaces.

Definition 2.8 Let $(F, \varsigma)$ be a $(D C M L S)$, and let $\left\{g_{n}\right\}_{n \geq 0}$ be a sequence in $F$.

(1) $\left\{g_{n}\right\}$ is convergent to $g$ in $F$ if and only if

$$
\lim _{n \rightarrow \infty} \varsigma\left(g_{n}, g\right)=\varsigma(g, g) .
$$

In this case, we write $\lim _{n \rightarrow \infty} g_{n}=g$.

(2) $\left\{g_{n}\right\}$ is $\varsigma$-Cauchy if and only if $\lim _{n, m \rightarrow \infty} \varsigma\left(g_{n}, g_{m}\right)$ exists and is finite.

(3) $(F, \varsigma)$ is said to be complete if for each $\varsigma$-Cauchy sequence $\left\{g_{n}\right\}$, there is $g \in F$ such that

$$
\lim _{n \rightarrow \infty} \varsigma\left(g_{n}, g\right)=\varsigma(g, g)=\lim _{n, m \rightarrow \infty} \varsigma\left(g_{n}, g_{m}\right)
$$

Definition 2.9 Let $(F, \varsigma)$ be a $(D C M L S)$. For $g \in F$ and $\tau>0$ :

(i) An open ball $\mathbb{B}(g, \tau)$ in $(F, \varsigma)$ is

$$
\mathbb{B}(g, \tau)=\{y \in F,|\varsigma(g, h)-\varsigma(g, g)|<\tau\} .
$$

(ii) The mapping $\aleph: F \rightarrow F$ is said to be continuous at $g \in F$ if for all $\varepsilon>0$, there exists $\delta>0$ such that $\zeta(\mathbb{B}(g, \delta)) \subseteq \mathbb{B}(\aleph(g), \varepsilon)$. Thus if $\aleph$ is continuous at $g$, then for any sequence $\left\{g_{n}\right\}$ converging to $g$, we have $\lim _{n \rightarrow \infty} \aleph g_{n}=\aleph g$, that is,

$$
\lim _{n \rightarrow \infty} \varsigma\left(\aleph g_{n}, \aleph g\right)=\varsigma(\aleph g, \aleph g)
$$

\section{Main results}

In our first theorem, we prove the Banach contraction type theorem in (DCMLS).

Theorem 3.1 Let $(\mathcal{F}, \varsigma)$ be a complete $(D C M L S)$ defined by functions $\varpi, \varepsilon: \mathcal{F}^{2} \rightarrow[1, \infty)$. Let $\aleph: \mathcal{F} \rightarrow \mathcal{F}$ be a mapping such that

$$
\varsigma(\aleph g, \aleph h) \leq k \varsigma(g, h)
$$

for all $g, h \in \mathcal{F}$, where $k \in(0,1)$. For $g_{0} \in \mathcal{F}$, take $g_{n}=\aleph^{n} g_{0}$. Suppose that

$$
\sup _{m \geq 1} \lim _{i \rightarrow \infty} \frac{\varpi\left(g_{i+1}, g_{i+2}\right)}{\varpi\left(g_{i}, g_{i+1}\right)} \varepsilon\left(g_{i+1}, g_{m}\right)<\frac{1}{k} .
$$

Also, assume that for every $g \in \mathcal{F}$, we have

$$
\lim _{n \rightarrow \infty} \varpi\left(g, g_{n}\right) \text { and } \lim _{n \rightarrow \infty} \varepsilon\left(g_{n}, g\right) \quad \text { exist and are finite. }
$$

Then $\aleph$ has a unique fixed point. 
Proof Let $\left\{g_{n}=\aleph^{n} g_{0}\right\}$ in $\mathcal{F}$ be a sequence that satisfies the conditions of our theorem. By using (3.1) we get

$$
\varsigma\left(g_{n}, g_{n+1}\right) \leq k^{n} \varsigma\left(g_{0}, g_{1}\right) \text { for all } n \geq 0 .
$$

Let $n, m \in \mathbb{N}$ be such that $n<m$. Then

$$
\begin{aligned}
& \varsigma\left(g_{n}, g_{m}\right) \leq \varpi\left(g_{n}, g_{n+1}\right) \varsigma\left(g_{n}, g_{n+1}\right)+\varepsilon\left(g_{n+1}, g_{m}\right) \varsigma\left(g_{n+1}, g_{m}\right) \\
& \leq \varpi\left(g_{n}, g_{n+1}\right) \varsigma\left(g_{n}, g_{n+1}\right)+\varepsilon\left(g_{n+1}, g_{m}\right) \varpi\left(g_{n+1}, g_{n+2}\right) \varsigma\left(g_{n+1}, g_{n+2}\right) \\
& +\varepsilon\left(g_{n+1}, g_{m}\right) \varepsilon\left(g_{n+2}, g_{m}\right) \varsigma\left(g_{n+2}, g_{m}\right) \\
& \leq \varpi\left(g_{n}, g_{n+1}\right) \varsigma\left(g_{n}, g_{n+1}\right)+\varepsilon\left(g_{n+1}, g_{m}\right) \varpi\left(g_{n+1}, g_{n+2}\right) \varsigma\left(g_{n+1}, g_{n+2}\right) \\
& +\varepsilon\left(g_{n+1}, g_{m}\right) \varepsilon\left(g_{n+2}, g_{m}\right) \varpi\left(g_{n+2}, g_{n+3}\right) \varsigma\left(g_{n+2}, g_{n+3}\right) \\
& +\varepsilon\left(g_{n+1}, g_{m}\right) \varepsilon\left(g_{n+2}, g_{m}\right) \varepsilon\left(g_{n+3}, g_{m}\right) \varsigma\left(g_{n+3}, g_{m}\right) \\
& \leq \cdots \\
& \leq \varpi\left(g_{n}, g_{n+1}\right) \varsigma\left(g_{n}, g_{n+1}\right)+\sum_{i=n+1}^{m-2}\left(\prod_{j=n+1}^{i} \varepsilon\left(g_{j}, g_{m}\right)\right) \varpi\left(g_{i}, g_{i+1}\right) \varsigma\left(g_{i}, g_{i+1}\right) \\
& +\prod_{k=n+1}^{m-1} \varepsilon\left(g_{k}, g_{m}\right) \varsigma\left(g_{m-1}, g_{m}\right) \\
& \leq \varpi\left(g_{n}, g_{n+1}\right) k^{n} \varsigma\left(g_{0}, g_{1}\right)+\sum_{i=n+1}^{m-2}\left(\prod_{j=n+1}^{i} \varepsilon\left(g_{j}, g_{m}\right)\right) \varpi\left(g_{i}, g_{i+1}\right) k^{i} \varsigma\left(g_{0}, g_{1}\right) \\
& +\prod_{i=n+1}^{m-1} \varepsilon\left(g_{i}, g_{m}\right) k^{m-1} \varsigma\left(g_{0}, g_{1}\right) \\
& \leq \varpi\left(g_{n}, g_{n+1}\right) k^{n} \varsigma\left(g_{0}, g_{1}\right)+\sum_{i=n+1}^{m-2}\left(\prod_{j=n+1}^{i} \varepsilon\left(g_{j}, g_{m}\right)\right) \varpi\left(g_{i}, g_{i+1}\right) k^{i} \varsigma\left(g_{0}, g_{1}\right) \\
& +\left(\prod_{i=n+1}^{m-1} \varepsilon\left(g_{i}, g_{m}\right)\right) k^{m-1} \varpi\left(g_{m-1}, g_{m}\right) \varsigma\left(g_{0}, g_{1}\right) \\
& =\varpi\left(g_{n}, g_{n+1}\right) k^{n} \varsigma\left(g_{0}, g_{1}\right)+\sum_{i=n+1}^{m-1}\left(\prod_{j=n+1}^{i} \varepsilon\left(g_{j}, g_{m}\right)\right) \varpi\left(g_{i}, g_{i+1}\right) k^{i} \varsigma\left(g_{0}, g_{1}\right) \\
& \leq \varpi\left(g_{n}, g_{n+1}\right) k^{n} \varsigma\left(g_{0}, g_{1}\right)+\sum_{i=n+1}^{m-1}\left(\prod_{j=0}^{i} \varepsilon\left(g_{j}, g_{m}\right)\right) \varpi\left(g_{i}, g_{i+1}\right) k^{i} \varsigma\left(g_{0}, g_{1}\right) \text {. }
\end{aligned}
$$

Note that we are using the fact that $\varpi(g, h) \geq 1$. Let

$$
\Omega_{p}=\sum_{i=0}^{p}\left(\prod_{j=0}^{i} \varepsilon\left(g_{j}, g_{m}\right)\right) \varpi\left(g_{i}, g_{i+1}\right) k^{i} .
$$


Then we have

$$
\varsigma\left(g_{n}, g_{m}\right) \leq \varsigma\left(g_{0}, g_{1}\right)\left[k^{n} \varpi\left(g_{n}, g_{n+1}\right)+\left(\Omega_{m-1}-\Omega_{n}\right)\right] .
$$

By condition (3.2), using the ratio test, we see that $\lim _{n \rightarrow \infty} \Omega_{n}$ exists, and hence the real sequence $\left\{\Omega_{n}\right\}$ is $\varsigma$-Cauchy. Finally, if we take the limit in inequality (3.5) as $n, m \rightarrow \infty$, we deduce that

$$
\lim _{n, m \rightarrow \infty} \varsigma\left(g_{n}, g_{m}\right)=0
$$

Hence the sequence $\left\{g_{n}\right\}$ is $\varsigma$-Cauchy in $(\mathcal{F}, \varsigma)$, which is a complete $(D C M L S)$, so $\left\{g_{n}\right\}$ converges to some $g \in \mathcal{F}$, that is,

$$
\lim _{n \rightarrow \infty} \varsigma\left(g_{n}, g\right)=\varsigma(g, g)=\lim _{n, m \rightarrow \infty} \varsigma\left(g_{n}, g_{m}\right)=0 .
$$

Then $\varsigma(g, g)=0$. Next, we show that $\aleph g=g$. The triangle inequality of $D C M L S$ implies that

$$
\varsigma\left(g, g_{n+1}\right) \leq \varpi\left(g, g_{n}\right) \varsigma\left(g, g_{n}\right)+\varepsilon\left(g_{n}, g_{n+1}\right) \varsigma\left(g_{n}, g_{n+1}\right) .
$$

Using (3.3) and (3.6), we deduce that

$$
\lim _{n \rightarrow \infty} \varsigma\left(g, g_{n+1}\right)=0 .
$$

By the triangle inequality and (3.1) we have

$$
\begin{aligned}
\varsigma(g, \aleph g) & \leq \varpi\left(g, g_{n+1}\right) \varsigma\left(g, g_{n+1}\right)+\varepsilon\left(g_{n+1}, \aleph g\right) \varsigma\left(g_{n+1}, \aleph g\right) \\
& \leq \varpi\left(g, g_{n+1}\right) \varsigma\left(g, g_{n+1}\right)+k \varepsilon\left(g_{n+1}, \aleph g\right) \varsigma\left(g_{n}, g\right) .
\end{aligned}
$$

Taking the limit as $n \rightarrow \infty$, by (3.3) and (3.8) we deduce that $\varsigma(g, \aleph g)=0$, that is, $\aleph g=g$. Finally, assume that $\aleph$ has two fixed points, say $\alpha$ and $\beta$. Then

$$
\varsigma(\alpha, \beta)=\varsigma(\aleph \alpha, \aleph \beta) \leq k \varsigma(\alpha, \beta)<\varsigma(\alpha, \beta)
$$

which leads us to a contradiction. Therefore $\varsigma(\alpha, \beta)=0$, so $\alpha=\beta$. Hence $\aleph$ has a unique fixed point.

Remark 3.2 Note that condition (3.3) in Theorem 3.1 can be changed by the assumption that $\aleph$ and the $(D C M L S) \varsigma$ are continuous. To see this, the continuity gives us that if $g_{n} \rightarrow$ $u$, then $\aleph g_{n} \rightarrow \aleph u$, and hence we have

$$
\lim _{n \rightarrow \infty} \varsigma\left(\aleph g_{n}, \aleph u\right)=0=\lim _{n \rightarrow \infty} \varsigma\left(\aleph g_{n+1}, \aleph u\right)=\varsigma(u, \aleph u),
$$

and thus $\aleph u=u$.

Now we illustrate Theorem 3.1 by the following example. 
Example 3.3 Consider $\mathcal{F}=\{0,1,2\}$. Let $\varsigma$ be symmetric and defined by

$$
\begin{aligned}
& \varsigma(g, g)=0 \quad \text { for } g \in\{0,1\}, \\
& \varsigma(2,2)=\frac{1}{100},
\end{aligned}
$$

and

$$
\varsigma(0,1)=1, \quad \varsigma(0,2)=\frac{2}{5}, \quad \varsigma(1,2)=\frac{6}{25} .
$$

Take $\varpi, \varepsilon: \mathcal{F} \times \mathcal{F} \rightarrow[1, \infty)$ to be symmetric and defined by

$$
\begin{aligned}
& \varpi(0,0)=\varpi(1,1)=\varpi(2,2)=1, \quad \varpi(0,2)=\frac{151}{100}, \\
& \varpi(1,2)=\frac{8}{5}, \quad \varpi(0,1)=\frac{6}{5},
\end{aligned}
$$

and

$$
\varepsilon(0,0)=\varepsilon(1,1)=\varepsilon(2,2)=1, \quad \varepsilon(0,2)=\frac{8}{5}, \quad \varepsilon(1,2)=\frac{33}{20}, \quad \varepsilon(0,1)=\frac{6}{5},
$$

Now define the self-mapping $\aleph$ on $\mathcal{F}$ as follows:

$$
\aleph 0=2 \text { and } \aleph 1=\aleph 2=1 \text {. }
$$

Choose $k=\frac{3}{5}$. Clearly, (3.1) holds. For any $g_{0} \in \mathcal{F}$, (3.2) holds, along with conditions of Theorem 3.1. Therefore the mapping $\aleph$ admits a unique fixed point, which is $g=1$.

Definition 3.4 Let $\aleph: \mathcal{F} \longrightarrow \mathcal{F}$. For some $g_{0} \in \mathcal{F}$, consider $O\left(g_{0}\right)=\left\{g_{0}, \aleph g_{0}, \aleph^{2} g_{0}, \ldots\right\}$ to be the orbit of $g_{0}$. We say that a function $G: \mathcal{F} \longrightarrow \mathbb{R}$ is $\aleph$-orbitally lower semicontinuous at $w \in \mathcal{F}$ if for $\left\{g_{n}\right\} \subset O\left(g_{0}\right)$ such that $g_{n} \longrightarrow w$, we have $G(w) \leq \lim _{n \rightarrow \infty} \inf G\left(g_{n}\right)$.

Inspired by [19], we are going to use Definition 3.4 to present a nice consequence of Theorem 3.1, which is a generalization of Theorem 1 in [22].

Corollary 3.5 Let $(\mathcal{F}, \varsigma)$ be the complete $(D C M L S)$ defined by functions $\varpi, \varepsilon: \mathcal{F}^{2} \rightarrow$ $[1, \infty)$. Let $\aleph: \mathcal{F} \rightarrow \mathcal{F}$, Let $g_{0} \in \mathcal{F}$ and $0<k<1$ be uch that

$$
\varsigma\left(\aleph w, \aleph^{2} w\right) \leq k \varsigma(w, \aleph w) \quad \text { for each } w \in O\left(g_{0}\right)
$$

Take $g_{n}=\aleph^{n} g_{0}$. Suppose that

$$
\sup _{m \geq 1} \lim _{i \rightarrow \infty} \frac{\varpi\left(g_{i+1}, g_{i+2}\right)}{\varpi\left(g_{i}, g_{i+1}\right)} \varepsilon\left(g_{i+1}, g_{m}\right)<\frac{1}{k} .
$$

Then $\lim _{n \rightarrow \infty} g_{n}=w \in \mathcal{F}$. Also, $\aleph w=w \Leftrightarrow g \mapsto \varsigma(g, \aleph g)$ is $\aleph$-orbitally lower semicontinuous at $w$.

Next, we present the nonlinear case. 
Theorem 3.6 Let $(\mathcal{F}, \varsigma)$ be a complete $(D C M L S)$ defined by functions $\varpi, \varepsilon: \mathcal{F}^{2} \rightarrow[1, \infty)$. Consider a map $\aleph: \mathcal{F} \rightarrow \mathcal{F}$ and assume that there exists a nondecreasing and continuous function $\phi: \mathbb{R}^{+} \rightarrow \mathbb{R}^{+}$such that

$$
\begin{aligned}
& \phi^{i}(g) \rightarrow 0 \quad \text { as } n \rightarrow \infty, g>0, \\
& \varsigma(\aleph g, \aleph h) \leq \phi(\Delta(g, h)), \quad \Delta(g, h)=\max \{\varsigma(g, h), \varsigma(g, \aleph g), \varsigma(h, \aleph h)\}
\end{aligned}
$$

for all $g, h \in \mathcal{F}$. Moreover, assume that for each $g_{0} \in \mathcal{F}$, we have

$$
\sup _{m \geq 1} \lim _{i \rightarrow \infty} \frac{\varpi\left(g_{i+1}, g_{i+2}\right)}{\varpi\left(g_{i}, g_{i+1}\right)} \varepsilon\left(g_{i+1}, g_{m}\right) \frac{\phi^{i+1}\left(\varsigma\left(g_{1}, g_{0}\right)\right)}{\phi^{i}\left(\varsigma\left(g_{1}, g_{0}\right)\right)}<1
$$

where $g_{n}=\aleph^{n} g_{0}, n \in \mathbb{N}$. If the $(D C M L S) \varsigma$ and $\aleph$ are continuous, then $\aleph$ admits a unique fixed point $w \in \mathcal{F}$ with $\aleph^{n} s \rightarrow$ w for each $g \in \mathcal{F}$.

Proof Let $\left\{g_{n}\right\}$ and $g_{0}$ be as in the hypothesis of the theorem. Assume that there exists $m \in \mathbb{N}$ such that $g_{m}=g_{m+1}=\aleph g_{m}$, which implies that $g_{m}$ is a fixed point. So we may assume that $g_{n+1} \neq g_{n}$ for each $n$. From condition (3.11) we have

$$
\varsigma\left(g_{n}, g_{n+1}\right)=\varsigma\left(\aleph g_{n}, \aleph g_{n-1}\right) \leq \phi\left(\Delta\left(g_{n-1}, g_{n}\right)\right),
$$

where $\Delta\left(g_{n-1}, g_{n}\right)=\max \left\{\varsigma\left(g_{n-1}, g_{n}\right), \varsigma\left(g_{n}, g_{n+1}\right)\right\}$. If for some $n$, we accept that $\Delta\left(g_{n-1}, g_{n}\right)=$ $\varsigma\left(g_{n}, g_{n+1}\right)$, then by (3.13) and the fact that we have $\phi(t)<t$ for all $t>0$, we deduce that

$$
0<\varsigma\left(g_{n}, g_{n+1}\right) \leq \phi\left(\varsigma\left(g_{n}, g_{n+1}\right)\right)<\varsigma\left(g_{n}, g_{n+1}\right)
$$

which is a contradiction. Thus, for all $n \in \mathbb{N}$, we obtain $\Delta\left(g_{n-1}, g_{n}\right)=\varsigma\left(g_{n-1}, g_{n}\right)$. It follows that $0<\varsigma\left(g_{n}, g_{n+1}\right) \leq \phi\left(\varsigma\left(g_{n-1}, g_{n}\right)\right)$. By using induction we easily see that for all $n \geq 0$,

$$
0<\varsigma\left(g_{n}, g_{n+1}\right) \leq \phi^{n}\left(\varsigma\left(g_{0}, g_{1}\right)\right) .
$$

By the properties of $\phi$ we can easily deduce that

$$
\varsigma\left(g_{n}, g_{n+1}\right) \rightarrow 0 \quad \text { as } n \rightarrow \infty .
$$

Using the argument in the proof of Theorem 3.1 , for $n, m \in \mathbb{N}$ such that $n<m$, we can easily deduce that

$$
\begin{aligned}
\varsigma\left(g_{n}, g_{m}\right) \leq & \varpi\left(g_{n}, g_{n+1}\right) \phi^{n}\left(\varsigma\left(g_{0}, g_{1}\right)\right) \\
& +\sum_{i=n+1}^{m-1}\left(\prod_{j=0}^{i} \varepsilon\left(g_{j}, g_{m}\right)\right) \varpi\left(g_{i}, g_{i+1}\right) \phi^{i}\left(\varsigma\left(g_{0}, g_{1}\right)\right) .
\end{aligned}
$$

By condition (3.12), using the ratio test, we can easily deduce that the sequence $\left\{g_{n}\right\}$ is $\varsigma$-Cauchy. Since $(\mathcal{F}, \varsigma)$ is a complete $(D C M L S)$, if $g_{n} \rightarrow w \in \mathcal{F}$ as $n \rightarrow \infty$, then 
$\lim _{n \rightarrow \infty} \varsigma\left(g_{n}, w\right)=0$. Hence by Remark 3.2 we conclude that $\aleph w=w$. Finally, assume that $w$ and $u$ are two fixed points of $\aleph$ such that $w \neq u$. From assumption (3.11) we have

$$
\varsigma(w, u)=\varsigma(\aleph w, \aleph u) \leq \phi(\Delta(w, u))=\phi(\varsigma(w, u))<\varsigma(w, u),
$$

which leads to a contradiction. Therefore $w=u$, as desired.

Remark 3.7 Note that if $\phi(g)=k g, 0<k<1$, then condition (3.11) in Theorem 3.6 becomes

$$
\varsigma(\aleph g, \aleph h) \leq k \max \{\varsigma(g, h), \varsigma(g, \aleph g), \varsigma(h, \aleph h)\} .
$$

Next, we prove the following result for mappings satisfying Kannan-type contraction.

Theorem 3.8 Let $(\mathcal{F}, \varsigma)$ be the complete $(D C M L S)$ defined by functions $\varpi, \varepsilon: \mathcal{F}^{2} \rightarrow$ $[1, \infty)$. Let $\aleph: \mathcal{F} \rightarrow \mathcal{F}$ be a Kannan mapping defined as follows:

$$
\varsigma(\aleph g, \aleph h) \leq a[\varsigma(g, \aleph g)+\varsigma(h, \aleph h)]
$$

for $g, h \in \mathcal{F}$, where $a \in\left(0, \frac{1}{2}\right)$. For $g_{0} \in \mathcal{F}$, take $g_{n}=\aleph^{n} g_{0}$. Suppose that

$$
\sup _{m \geq 1} \lim _{i \rightarrow \infty} \frac{\varpi\left(g_{i+1}, g_{i+2}\right)}{\varpi\left(g_{i}, g_{i+1}\right)} \varepsilon\left(g_{i+1}, g_{m}\right)<\frac{1-a}{a} .
$$

Also, assume that for every $g \in \mathcal{F}$, we have

$$
\lim _{n \rightarrow \infty} \varpi\left(g, g_{n}\right) \quad \text { exists and is finite, and } \lim _{n \rightarrow \infty} \varepsilon\left(g_{n}, g\right)<\frac{1}{a} \text {. }
$$

Then $\aleph$ has a fixed point. Moreover, if for every fixed point $w$, we have $\varsigma(w, w)=0$, then the fixed point is unique.

Proof Consider the sequence $\left\{g_{n}=\aleph g_{n-1}\right\}$ in $\mathcal{F}$ satisfying hypotheses (3.18) and (3.19). From (3.17) we obtain

$$
\begin{aligned}
\varsigma\left(g_{n}, g_{n+1}\right) & =\varsigma\left(\aleph g_{n-1}, \aleph g_{n}\right) \\
& \leq a\left[\varsigma\left(g_{n-1}, \aleph g_{n-1}\right)+\varsigma\left(g_{n}, \aleph g_{n}\right)\right] \\
& =a\left[\varsigma\left(g_{n-1}, g_{n}\right)+\varsigma\left(g_{n}, g_{n+1}\right)\right] .
\end{aligned}
$$

Then $\varsigma\left(g_{n}, g_{n+1}\right) \leq \frac{a}{1-a} \varsigma\left(g_{n-1}, g_{n}\right)$. By induction we get

$$
\varsigma\left(g_{n}, g_{n+1}\right) \leq\left(\frac{a}{1-a}\right)^{n} \varsigma\left(g_{1}, g_{0}\right), \quad \forall n \geq 0 .
$$

Next, we show that $\left\{g_{n}\right\}$ is a $\varsigma$-Cauchy sequence. For two natural numbers $n<m$, we have

$$
\varsigma\left(g_{n}, g_{m}\right) \leq \varpi\left(g_{n}, g_{n+1}\right) \varsigma\left(g_{n}, g_{n+1}\right)+\varepsilon\left(g_{n+1}, g_{m}\right) \varsigma\left(g_{n+1}, g_{m}\right) .
$$


Similarly to the proof of Theorem 3.1, we get

$$
\begin{aligned}
\varsigma\left(g_{n}, g_{m}\right) \leq & \varpi\left(g_{n}, g_{n+1}\right) \varsigma\left(g_{n}, g_{n+1}\right)+\sum_{i=n+1}^{m-2}\left(\prod_{j=n+1}^{i} \varepsilon\left(g_{j}, g_{m}\right)\right) \varpi\left(g_{i}, g_{i+1}\right) \varsigma\left(g_{i}, g_{i+1}\right) \\
& +\prod_{k=n+1}^{m-1} \varepsilon\left(g_{k}, g_{m}\right) \varsigma\left(g_{m-1}, g_{m}\right) \\
\leq & \varpi\left(g_{n}, g_{n+1}\right)\left(\frac{a}{1-a}\right)^{n} \varsigma\left(g_{0}, g_{1}\right) \\
& +\sum_{i=n+1}^{m-2}\left(\prod_{j=n+1}^{i} \varepsilon\left(g_{j}, g_{m}\right)\right) \varpi\left(g_{i}, g_{i+1}\right)\left(\frac{a}{1-a}\right)^{i} \varsigma\left(g_{0}, g_{1}\right) \\
& +\prod_{i=n+1}^{m-1} \varepsilon\left(g_{i}, g_{m}\right)\left(\frac{a}{1-a}\right)^{m-1} \varsigma\left(g_{0}, g_{1}\right) .
\end{aligned}
$$

Since $0 \leq a<\frac{1}{2}$, we have $0<\frac{a}{1-a}<1$, and similarly to the argument in the proof of Theorem 3.1, we obtain that $\left\{g_{n}\right\}$ is a $\varsigma$-Cauchy sequence in the complete $(D C M L S)(\mathcal{F}, \varsigma)$. Thus $\left\{g_{n}\right\}$ converges to some $w \in \mathcal{F}$. Suppose that $\aleph w \neq w$. Then

$$
\begin{aligned}
0<\varsigma(w, \aleph w) & \leq \varpi\left(w, g_{n+1}\right) \varsigma\left(w, g_{n+1}\right)+\varepsilon\left(g_{n+1}, \aleph w\right) \varsigma\left(g_{n+1}, \aleph w\right) \\
& \leq \varpi\left(w, g_{n+1}\right) \varsigma\left(w, g_{n+1}\right)+\varepsilon\left(g_{n+1}, \aleph w\right)\left[a \varsigma\left(g_{n}, g_{n+1}\right)+a \varsigma(w, \aleph w)\right] .
\end{aligned}
$$

Taking the limit in both sides of these inequalities and using (3.19), we deduce that $0<$ $\varsigma(w, \aleph w)<\varsigma(w, \aleph w)$, which is a contradiction. Hence $\aleph w=w$. Now assume that for every fixed point $w$, we have $\varsigma(w, w)=0$ and suppose that $\aleph$ has more than one fixed point, say $w$ and $\lambda$. Then

$$
\begin{aligned}
\varsigma(w, \lambda)=\varsigma(\aleph w, \aleph \lambda) & \leq a[\varsigma(w, \aleph w)+\varsigma(\lambda, \aleph \lambda)] \\
& =a[\varsigma(w, w)+\varsigma(\lambda, \lambda)]=0 .
\end{aligned}
$$

Thereby $w=\lambda$, as required.

\section{Conclusion}

In this section, we present an open question on $(D C M L S)$ endowed with a graph. First, Fig. 1 is an example of a $(D C M L S)$ endowed with a graph.

Let $\varsigma$ be a $(D C M L S)$ on a set $\mathcal{F} \neq \emptyset$. Let $\Delta$ be the diagonal of $\mathcal{F}^{2}$. A graph $G$ is defined by the pair $(V, E)$, where $V$ is a set of vertices coinciding with $\mathcal{F}$, and $E$ is the set of its edges with $\Delta \subset E$. From now on, assume that $G$ has no parallel edges.

Definition 4.1 Let $t$ and $g$ be two vertices in a graph $G$. A path in $G$ from $t$ to $g$ of length $q(q \in \mathbb{N} \cup\{0\})$ is a sequence $\left(k_{i}\right)_{i=0}^{q}$ of $q+1$ distinct vertices such that $k_{0}=t, k_{n}=g$, and $\left(k_{i}, k_{i+1}\right) \in E(G)$ for $i=1,2, \ldots, q$.

The graph $G$ may be converted to a weighted graph by assigning to each edge the distance given by the (DCMLS) between its vertices. 


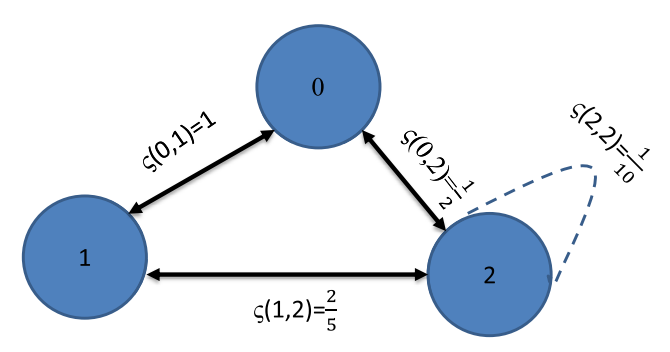

Figure $1(D C M L S)$ as in Example 2.7

Notation Let $\mathcal{F}^{\aleph}=\{x \in \mathcal{F} /(x, \aleph x) \in E(G)$ or $(\aleph x, x) \in E(G)\}$.

Definition 4.2 Let $(\mathcal{F}, \varsigma)$ be a complete $(D C M L S)$ endowed with a graph $G$. The mapping $\aleph: \mathcal{F} \rightarrow \mathcal{F}$ is said to be a $G_{\phi}$-contraction if

$$
\text { for all } t, g \in \mathcal{F}, \quad(t, g) \in E(G) \Longrightarrow(\aleph t, \aleph g) \in E(G)
$$

- there is a function $\phi: \mathbb{R}^{+} \longrightarrow \mathbb{R}^{+}$such that

$$
\xi\left(\aleph t, \aleph^{2} t\right) \leq \phi(\xi(t, \aleph t)), \quad \forall t \in \mathcal{F}^{\aleph}
$$

where $\phi$ is a nondecreasing function, and $\left\{\phi^{n}(t)\right\}_{n \in \mathbb{N}}$ converges to 0 for each $t>0$.

Definition 4.3 The mapping $\aleph: \mathcal{F} \longrightarrow \mathcal{F}$ is called orbitally $G$-continuous if for all $a, b \in X$ and any positive sequence $\left\{g_{n}\right\}_{n \in \mathbb{N}}$,

$$
\aleph^{g_{n}} a \longrightarrow b,\left(\aleph^{g_{n}} a, \aleph^{g_{n+1}} a\right) \in E(G) \Longrightarrow \aleph\left(\aleph^{g_{n}} a\right) \longrightarrow \aleph b \quad \text { as } n \rightarrow \infty
$$

Conjecture 4.4 Let $(\mathcal{F}, \varsigma, G)$ be a complete $(D C M L S)$ with a graph $G$. Let $\aleph: \mathcal{F} \rightarrow \mathcal{F}$ be a $G_{\phi}$-contraction that is orbitally $G$-continuous. Suppose the following property holds:

(P) for any $\left\{t_{n}\right\}_{n \in \mathbb{N}}$ in $\mathcal{F}$, if $t_{n} \longrightarrow t$ and $\left(t_{n}, t_{n+1}\right) \in E(G)$, then there is a subsequence $\left\{t_{k_{n}}\right\}_{n \in \mathbb{N}}$ with $\left(t_{k_{n}}, t\right) \in E(G)$.

Moreover, suppose that, for each $g \in \mathcal{F}$,

$$
\sup _{m \geq 1} \lim _{i \rightarrow \infty} \frac{\varpi\left(g_{i+1}, g_{i+2}\right)}{\varpi\left(g_{i}, g_{i+1}\right)} \varepsilon\left(g_{i+1}, g_{m}\right)<M ; \quad M>1 .
$$

Also, assume that for every $g \in \mathcal{F}$, we have that

$$
\lim _{n \rightarrow \infty} \varpi\left(g, g_{n}\right) \text { and } \lim _{n \rightarrow \infty} \varepsilon\left(g_{n}, g\right) \quad \text { exist and are finite. }
$$

Then the restriction of $\aleph_{\mid[g]_{\tilde{G}}}$ to $[g]_{\tilde{G}}$ possesses a fixed point. 
Funding

Not applicable.

\section{Availability of data and materials}

Not applicable.

\section{Competing interests}

The author declares no conflict of interest.

Authors' contributions

The author read and approved the final manuscript.

\section{Publisher's Note}

Springer Nature remains neutral with regard to jurisdictional claims in published maps and institutional affiliations.

\section{Received: 7 April 2020 Accepted: 8 July 2020 Published online: 17 July 2020}

\section{References}

1. Banach, S.: Sur les opérations dans les ensembles abstraits et leur applications aux équations intègrales. Fundam. Math. 3(1), 133-181 (1922)

2. Abdeljawad, T., Abodayeh, K., Mlaiki, N.: On fixed point generalizations to partial $b$-metric spaces. J. Comput. Anal. Appl. 19(5), 883-891 (2015)

3. Rashwan, R.A., Mahmoud, M.G.: Common fixed point theorems for weakly compatible self-mappings under contraction conditions in complex valued $b$-metric spaces. Palest. J. Math. 9(2), 749-760 (2020)

4. Qawaqneh, H., Noorani, M.S.M., Shatanawi, W., Aydi, H., Alsamir, H.: Fixed point results for multi-valued contractions in b-metric spaces and an application. Mathematics 7(2), Article ID 132 (2019)

5. Shatanawi, W., Abodayeh, K., Mukhemer, A.: Some fixed point theorems in extended $b$-metric spaces. UPB Sci. Bull., Ser. A, Appl. Math. Phys. 80(4), 71-78 (2018)

6. Shatanawi, W., Pitea, A., Lazovic, R.: Contraction conditions using comparison functions on $b$-metric spaces. Fixed Point Theory Appl. 2014(1), Article ID 135 (2014)

7. Afshari, H., Atapour, M., Aydi, H.: Generalized $\alpha-\psi$-Geraghty multivalued mappings on $b$-metric spaces endowed with a graph. TWMS J. Appl. Eng. Math. 7(2), 248-260 (2017)

8. Alharbi, N., Aydi, H., Felhi, A., Ozel, C., Sahmim, S.: $\alpha$-Contractive mappings on rectangular $b$-metric spaces and an application to integral equations. J. Math. Anal. 9(3), 47-60 (2018)

9. Aydi, H., Karapinar, E., Bota, M.F., Mitrović, S.: A fixed point theorem for set-valued quasi-contractions in b-metric spaces. Fixed Point Theory Appl. 2012, Article ID 88 (2012)

10. Aydi, H., Bota, M.F., Karapinar, E., Moradi, S.: A common fixed point for weak $\phi$-contractions on $b$-metric spaces. Fixed Point Theory 13(2), 337-346 (2012)

11. Aydi, H., Banković, R., Mitrović, I., Nazam, M.: Nemytzki-Edelstein-Meir-Keeler type results in b-metric spaces. Discrete Dyn. Nat. Soc. 2018, Article ID 4745764 (2018)

12. Czerwik, S.: Contraction mappings in b-metric spaces. Acta Math. Inform. Univ. Ostrav. 1, 5-11 (1993)

13. Mlaiki, N., Mukheimer, A., Rohen, Y., Souayah, N., Abdeljawad, T.: Fixed point theorems for $\alpha-\psi$-contractive mapping in $\Omega_{b}$-metric spaces. J. Math. Anal. 8(5), 40-46 (2017)

14. Souayah, N., Mlaiki, N., Mrad, M.: The $G_{M}$-contraction principle for mappings on $M$-metric spaces endowed with a graph and fixed point theorems. IEEE Access 6, 25178-25184 (2018)

15. Souayah, N., Mlaiki, N.: A fixed point theorem in $S_{6}$ metric spaces. J. Math. Comput. Sci. 16, 131-139 (2016)

16. Alamgir, N., Kiran, Q., Isık, H., Aydi, H.: Fixed point results via a Hausdorff controlled type metric. Adv. Differ. Equ. 2020, Article ID 24 (2020)

17. Kiran, Q., Alamgir, N., Mlaiki, N., Aydi, H.: On some new fixed point results in complete extended b-metric spaces. Mathematics 7(5), Article ID 476 (2019)

18. Bakhtin, I.A.: The contraction mapping principle in almost metric spaces. Funct. Anal. 30, 26-37 (1989)

19. Kamran, T., Samreen, M., UI Ain, Q.: A generalization of $b$-metric space and some fixed point theorems. Mathematics 5, Article ID 19 (2017)

20. Mlaiki, N., Aydi, H., Souayah, N., Abdeljawad, T.: Controlled metric type spaces and the related contraction principle. Mathematics 6(10), Article ID 194 (2018)

21. Abdeljawad, T., Mlaiki, N., Aydi, H., Souayah, N.: Double controlled metric type spaces and some fixed point results, Mathematics 6, Article ID 320 (2018). https://doi.org/10.3390/math6120320

22. Hicks, T.L., Rhodes, B.E.: A Banach type fixed point theorem. Math. Jpn. 24, 327-330 (1979) 